\title{
Correlation of chromosomal imbalances by comparative genomic hybridization and expression of EGFR, PTEN, p53, and MIB-1 in diffuse gliomas
}

\author{
TAHSIN YAKUT ${ }^{1}$, ANGELIKA GUTENBERG $^{2}$, AHMET BEKAR $^{3}$, UNAL EGELI $^{4}$, BASTIAN GUNAWAN $^{5}$, \\ ILKER ERCAN $^{6}$, SAHSENE TOLUNAY $^{7}$, MUAMMER DOYGUN $^{4}$ and HANS-JURGEN SCHULTEN ${ }^{5}$ \\ ${ }^{1}$ Medical Genetics Department, Faculty of Medicine, Uludag University, Bursa, Turkey; ${ }^{2}$ Department for \\ Neurosurgery, University of Göttingen, Germany; Departments of ${ }^{3}$ Neurosurgery and ${ }^{4}$ Medical Biology, \\ Faculty of Medicine, Uludag University, Bursa, Turkey; ${ }^{5}$ Institute of Pathology, University of Göttingen, \\ Germany; Departments of ${ }^{6}$ Biostatistics and ${ }^{7}$ Pathology, Faculty of Medicine, Uludag University, Bursa, Turkey
}

Received November 18, 2006; Accepted December 29, 2006

\begin{abstract}
The histological subclassification of gliomas is increasingly assisted by the underlying molecular genetics which has major importance in guiding clinical management of the disease. However, the assessment of several molecular events for improving clinical care remains a challenge. Herein, we report on comparative genomic hybridization (CGH) and immunohistochemical (IHC) assessment of EGFR, PTEN, p53, and MIB-1 expression in 13 oligodendrogliomas (10 WHO grade II, 3 WHO grade III), one oligoastrocytoma (WHO grade III) and 23 high-grade astrocytomas (3 WHO grade III, 20 glioblastoma multiforme). The most frequent imbalances in oligodendroglial tumors including the oligoastrocytic case were, in decreasing order of frequency, $+7 q,-1 p$, and $-4 q$ and in astrocytomas $+7 \mathrm{q},-10 \mathrm{q},+7 \mathrm{p},-9 \mathrm{p},-10 \mathrm{p},+20 \mathrm{q}$, and $+20 \mathrm{p}$. Some individual imbalances were associated with increasing numbers of chromosomal changes, that were $+7 q$ in both oligodendrogliomas and astrocytomas, and $-9 \mathrm{p},-10 \mathrm{q},+20 \mathrm{p}$, and $+20 \mathrm{q}$ in astrocytomas. The markers p53 and MIB-1 were significantly higher expressed in astrocytomas than in oligodendrogliomas and expression levels of p53 and EGFR were inversely associated within the astrocytic group. In addition, p53 overexpression correlated positively with $+7 \mathrm{q}$ and negatively with $-1 \mathrm{p}$ in the oligodendroglial group whereas EGFR overexpression correlated positively with $-1 p$ in the oligodendroglial and positively with $+7 \mathrm{p}$ and $-10 \mathrm{p}$ in the astrocytic group. Short overall survival was significantly
\end{abstract}

Correspondence to: Dr Tahsin Yakut, Medical Genetics Department, Faculty of Medicine, Uludag University, 16059 Bursa, Turkey

E-mail: tyakut@uludag.edu.tr

Key words: comparative genomic hybridization, immunohistochemistry, oligodendrogliomas, astrocytomas associated with $+7 p$ and $-10 q$ in astrocytomas. Collectively, these results contribute to the increasing clinical relevance of assessing tumor biological markers in gliomas.

\section{Introduction}

Diffuse gliomas are the most common primary central nervous tumors in adults and are histopathologically classified as oligodendrogliomas, astrocytomas, and mixed oligoastrocytomas. The WHO (World Health Organisation) grading system defines the spectrum from low to high grade gliomas comprising in the astrocytic lineage a wide range of genetically and clinically distinct tumors from grade I pilocytic astrocytomas, grade II diffuse astrocytomas, grade III anaplastic astrocytomas to grade IV glioblastoma multiforme (GBM) (1). The clinical management of gliomas remains a challenge, because of their infiltrative pattern of invasion, complete resection of all glial tumor components is almost impossible and adjuvant treatment still does not improve survival in most patients with the exception of pilocytic astrocytomas which can be successfully treated by complete tumor resection $(2,3)$. Molecular genetic analysis has added useful information regarding the characteristic pattern of aberrations for the different histological subtypes. Oligodendrogliomas are commonly characterized by losses of $1 p$ and 19q whereas astrocytomas have commonly gains of the entire or part of chromosome 7 and loss of 10 . The importance of distinguishing oligodendrogliomas from astrocytomas depends on the predicted sensitivity to chemotherapy of oligodendrogliomas marked with $1 \mathrm{p}$ loss or combined $1 \mathrm{p} / 19 \mathrm{q}$ losses (4). Other aberrations frequently occurring in both histological subtypes include gains at $8 \mathrm{q}$, $12 \mathrm{q}$ and losses at $9 \mathrm{p}$ harbouring the known oncogenes $M Y C$, $C D K 4$ and the tumor suppressor genes $C D K N 2 A$ and $C D K N 2 B$ (5). This summary report by Koschny and colleagues on $>500$ gliomas also demonstrated that the average number of copy alterations correlates with malignancy in oligodendrogliomas as well as in astrocytomas. Recent advances in molecular genetic approaches have led to the identification of cellular molecules and pathways severely affected in gliomas 
and contributed thus to the understanding of the biological behaviour of gliomas (6). Epidermal growth factor receptor (EGFR) gene located on chromosome 7 p11.2 is involved in controlling cell proliferation and is frequently overexpressed or mutated in astrocytomas leading to tumor progression, decreased apoptosis and tumor cell invasion. Gains or amplifications of chromosome 7 seem to be closely related with EGFR-immunoreactive tumor cells in gliomas (7). The phosphatase and tensine homologous gene PTEN, located on 10q23.3 acts as a phosphatidylinositol 3,4,5-triphosphate phosphatase and its tumor suppressor function is impaired in a variety of malignancies. PTEN is frequently affected by mutations in the coding region and by loss of heterozygosity $(\mathrm{LOH})$ or structural rearrangements of chromosome 10 in gliomas (8). PTEN controls cell proliferation via the PI3 kinase/AKT pathway which becomes deregulated owing to PTEN inactivation (6). Immunohistochemical expression of PTEN has been shown to correlate with better prognosis (9). p53 acts under normal conditions as a tumor suppressor by its antimitotic quality. It is frequently mutated in gliomas which leads to abolished tumor suppressor function and to nuclear accumulation of the protein, which in turn is accessible to nuclear staining with p53 antibodies. The impact of p53 mutation on the prognosis of gliomas is subject of controversy (3) and it seems that p53 mutations are more related to secondary than to primary gliomas (10).

The aim of the present study was to evaluate genetic imbalances by $\mathrm{CGH}$ and immunohistochemistry in diffuse gliomas and comparing the results with clinicopathological features in order to contribute to an improved clinical management of gliomas.

\section{Materials and methods}

Patients and tumor specimens. Tumor tissue samples of 37 human glioma specimens were studied from patients who underwent neurosurgery at the Medical Faculty of Uludag University. The tumor specimens were collected between September 1997 and July 2003. Hematoxylin and eosin-stained sections from each specimen were assessed by neuropathologists (A.G., S.T.) according to the WHO classification system (1).

Comparative genomic hybridization. Genomic tumor DNA was isolated from snap-frozen tumor specimens in 26 cases and from dewaxed, formalin-fixed and paraffin-embedded specimens in 11 cases by proteinase $\mathrm{K}$ digestion and spin column purification (Qiagen, Hilden, Germany). CGH was performed as described previously (11). Briefly, labelling of tumor DNA with biotin-16-dUTP (Roche, Mannheim, Germany) and normal reference DNA with digoxigenin-11dUTP (Roche) was performed by standard nick translation. The denatured DNA probes containing each 1.5 to $2 \mu \mathrm{g}$ of tumor DNA and reference DNA and $80 \mu \mathrm{g}$ of COT-1 DNA were hybridized for 3 days to normal metaphase spreads (Vysis, Downers Grove, IL). Subsequently, the slides were washed extensively, blocked with bovine serum albumin solution and incubated with fluorescein-conjugated avidin (Vector Laboratories, Burlingame, CA) and rhodamineconjugated antidigoxigenin (Roche). The slides were then
A

\begin{tabular}{|c|c|c|c|c|}
\hline 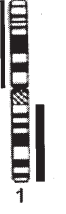 & $\begin{array}{c}\text { 旧 } \\
\text { 盟 } \\
\text { 目 } \\
\text { 目 } \\
2\end{array}$ & 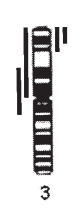 & 、㮌 & $\begin{array}{l}\text { 厡 } \\
\text { 亶 } \\
5\end{array}$ \\
\hline 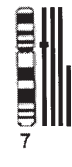 & 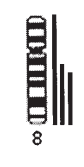 & 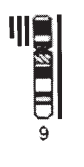 & 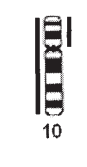 & 监 \\
\hline 叠 & 盽 $_{14}$ & 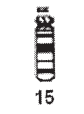 & $\begin{array}{l}\text { 貣 } \\
16 \\
16\end{array}$ & $\underset{17}{\Xi_{1}}$ \\
\hline 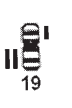 & $\underset{20}{\text { 이 }^{\prime}}$ & $\begin{array}{l}\stackrel{\mathbb{B}}{*}_{1} \\
21\end{array}$ & $\begin{array}{l}\stackrel{\mathrm{m}}{\mathrm{B}} \\
22\end{array}$ & $\|$ \\
\hline
\end{tabular}

B

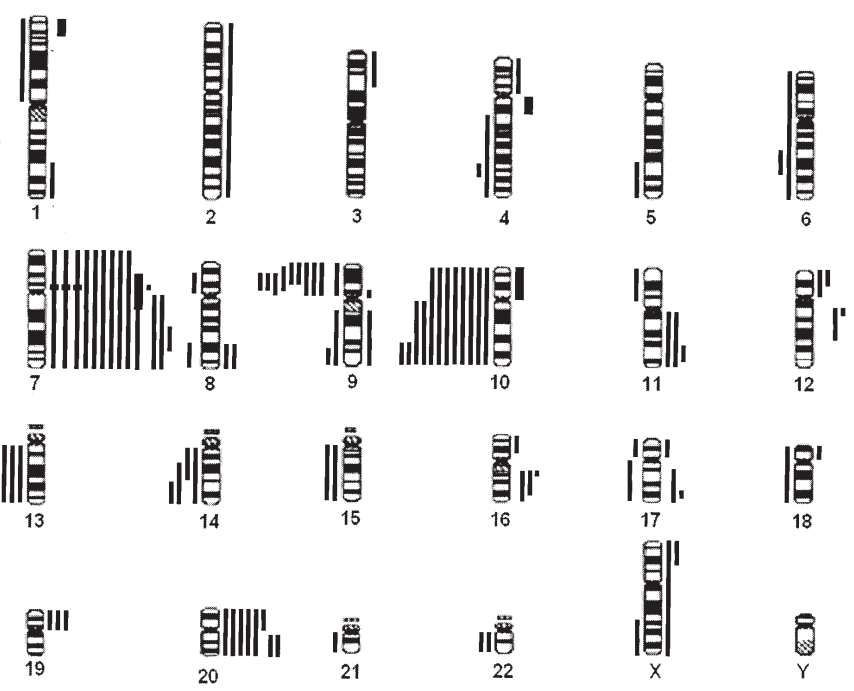

Figure 1. Overview of chromosomal imbalances in 14 oligodendroglial (A) and 23 high-grade astrocytic tumors (B). Bars on the left of each ideogram represent losses and on the right gains, corresponding to the chromosomal regions. Amplifications are in bold.

washed again and finally mounted in an antifade medium (vectashield, Vector Laboratories) supplemented with actinomycin $(12.5 \mu \mathrm{g} / \mathrm{ml})$ and DAPI (4',6-diamidino-2-phenylindole) $(1.25 \mu \mathrm{g} / \mathrm{ml})$ for counterstain. Image acquisition was performed on a Zeiss Axioskop fluorescence microscope (Zeiss, Göttingen, Germany) equipped with three separate bandpass filters for DAPI, green, and red fluorescence spectrum and a high sensitive monochrome charge coupled device camera (Photometrics, Tucson, AZ). For each analysis, the averaged chromosome-specific green to red fluorescence ratios, from at least 10 well selected metaphases, were plotted using the Quips CGH software (distributed through Applied Imaging, Newcastle, UK). Relative copy number changes were interpreted as gains when the average green-to-red ratio exceeded 1.15 (1.5 for amplifications) and as losses when the 


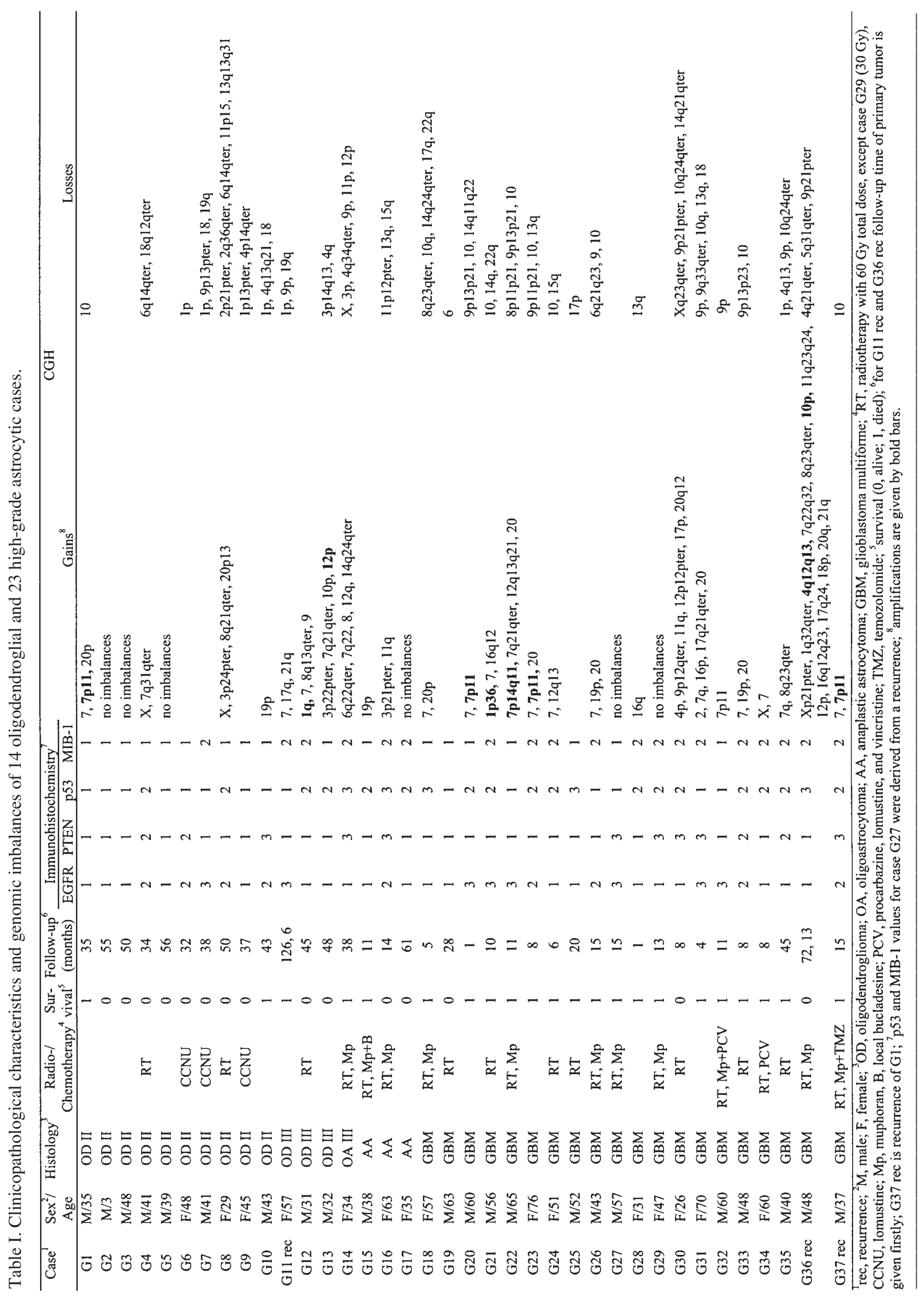


(A)

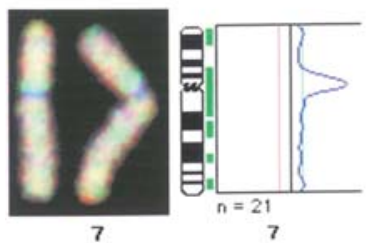

(B)

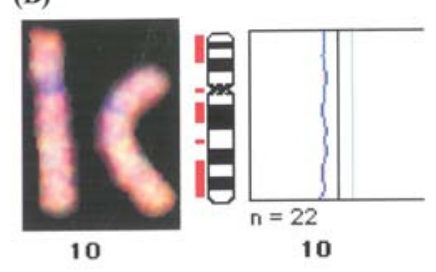

Figure 2. Example of chromosomal imbalances in GBM shown by a representative fluorescent karyogram and corresponding ideogram of case G22 for chromosome 7 (A) and $10(\mathrm{~B})$. This case is marked by gains/amplifications at $7 \mathrm{p}, 7 \mathrm{q}, 12 \mathrm{q}$, and 20 as well as losses at $8 \mathrm{p}, 9 \mathrm{p}$, and 10 . The ideogram is shown along with its chromosome-specific green-to-red fluorescent ratio profiles and bars to the right represent gains/amplifications and bars to the left represent losses ( $\mathrm{n}$ indicates number of analyzed chromosomes).

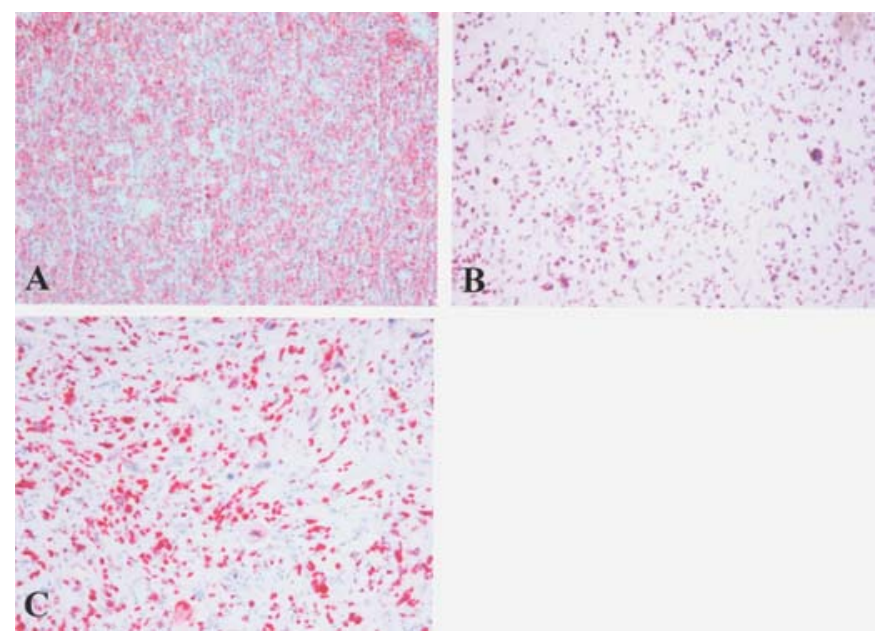

Figure 3. EGFR overexpression in case G21 (score 3) (A), PTEN overexpression in case G16 (score 3) (B), and p53 overexpression in case G18 (score 3) (C). Magnification, x200.

corresponding ratio was $<0.85$. Exceptionally, in cases with only trends not reaching the aforementioned thresholds, deviations from normal were classified as gains or losses when the $95 \%$ confidence interval varied beyond the ratio of 1.0 . Chromosomal regions 13p, 14p, 15p, 21p, 22p, Y, telomeres and constitutive heterochromatic regions at $1 \mathrm{q}, 9 \mathrm{q}$, and $16 \mathrm{q}$ known to produce false results by $\mathrm{CGH}$ were excluded from all analyses (11).

Immunohistochemistry. IHC evaluations were performed for p53 (mouse monoclonal, clone DO-7, dilution 1:50, microwave pre-treatment; Dako, Hamburg, Germany), PTEN (mouse monoclonal, clone 28H6, dilution 1:50, microwave pretreatment; NeoMarkers, Fremont, CA, USA), EGFR (mouse monoclonal, clone E30, dilution 1:1500, protease pretreatment; Dako), and the Ki-67 antigen (Clone MIB-1, dilution 1:50, microwave pre-treatment; Dako) using the DAK ChemMate $^{\mathrm{TM}}$ detection kit (Dako) for visualization. Levels of p53, PTEN and EGFR staining were expressed as the product of labelling index (LI) and percentage of positivestained tumor cells from 10 high power random fields. The labelling index was scored into categories, $1(<10 \%$, no or minimal reactivity corresponding with normal brain tissue

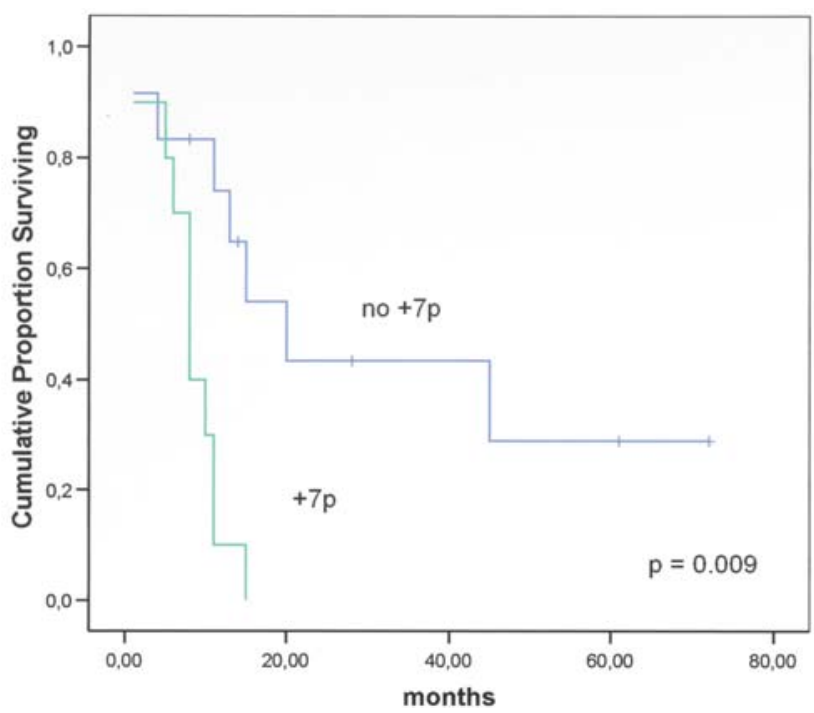

Figure 4. Kaplan-Meier plot of survival rates revealing worse prognosis of astrocytic cases with $+7 \mathrm{p}$ compared with cases showing no $+7 \mathrm{p}$. Case G37 rec, the recurrence of $\mathrm{G} 1$, was excluded from this analysis.

reactivity), $2(10=\mathrm{LI}<50 \%)$, or $3(\mathrm{LI}=50 \%)$. For p53 and PTEN only nuclear staining and for EGFR only membranous staining was considered. The MIB-1 score was derived from the percentage of positive-stained tumor cells in 10 high power fields and categorized into $1(=5 \%)$ or $2(>5 \%)$.

Statistical analysis. Individual imbalances were defined as gain, including amplification, or loss involving a chromosome arm. The Fisher exact test for contingency tables, Wilcoxon sum test and Kruskal-Wallis test were used to evaluate significant differences in frequencies of most common chromosomal imbalances and clinicopathological factors including IHC data. The association of the most common individual imbalances and clinicopathological factors with overall survival rates was tested with the log-rank test for censored data and visualized using the Kaplan-Meier method. The $p$-values for accepting significance $(p=0.05)$ were not adjusted for multiple comparisons. Statistical analysis was performed using the SPSS program (SPSS, Chicago, IL, USA).

\section{Results}

Clinicopathological and follow-up data. Thirty-seven glial cases were incorporated in this study comprising 13 oligodendrogliomas (10 WHO grade II; 3 WHO grade III), one oligoastrocytoma (WHO grade III) and 23 high-grade astrocytomas (3 anaplastic astrocytomas, WHO grade III; 20 GBM, WHO grade IV). In the oligodendroglial group including the oligoastrocytic case, nine patients were male and 5 female (mean age, 37.6 years, \pm 12.6 ) and in the astrocytic group 13 patients were male and 10 female (mean age, 51.4 years, \pm 12.9 ). Thirty-four were primary and 3 were recurrent tumors, altogether obtained from 36 patients. Seven of 11 oligodendroglial and 18 of 23 astrocytic cases received either radio- and/or chemotherapy which in all instances was applied postoperatively. Mean follow-up time was 49.1 months (range, 32-126 months, \pm 18.3 ) in the oligodendroglial and 
Table II. Correlation of chromosomal imbalances and clinicopathological features.

\begin{tabular}{|c|c|c|c|}
\hline Variables & Group $^{a}$ & p-value & Test $^{\mathrm{b}}$ \\
\hline \multicolumn{4}{|l|}{$\begin{array}{l}\text { Overall imbalances vs. } \\
\text { individual imbalances }\end{array}$} \\
\hline Overall changes vs. $+7 q$ & OD & 0.026 & WT \\
\hline Overall changes vs. $+7 q$ & $\mathrm{AC}$ & 0.001 & WT \\
\hline Overall changes vs. $-9 p$ & $\mathrm{AC}$ & 0.001 & WT \\
\hline Overall changes vs. $-10 q$ & $\mathrm{AC}$ & 0.001 & WT \\
\hline Overall changes vs. $+20 p$ & $\mathrm{AC}$ & 0.007 & WT \\
\hline Overall changes vs. $+20 q$ & $\mathrm{AC}$ & $<0.001$ & WT \\
\hline \multicolumn{4}{|l|}{$\begin{array}{l}\text { Individual imbalances vs. } \\
\text { individual imbalances }\end{array}$} \\
\hline$+7 p$ vs. $+7 q$ & $\mathrm{AC}$ & 0.003 & FET \\
\hline$+7 p$ vs. $-10 p$ & $\mathrm{AC}$ & $<0.001$ & FET \\
\hline$+7 p$ vs. $-10 q$ & $\mathrm{AC}$ & 0.012 & FET \\
\hline$+7 q$ vs. $-10 p$ & $\mathrm{AC}$ & 0.006 & FET \\
\hline$+7 q$ vs. $-10 q$ & $\mathrm{AC}$ & 0.001 & FET \\
\hline$+7 \mathrm{q}$ vs. $+20 \mathrm{p}$ & $\mathrm{AC}$ & 0.019 & FET \\
\hline$-9 p$ vs. $-10 q$ & $\mathrm{AC}$ & 0.036 & FET \\
\hline$-9 p$ vs. $+20 q$ & $\mathrm{AC}$ & $<0.001$ & FET \\
\hline$-10 p$ vs. $-10 q$ & $\mathrm{AC}$ & 0.001 & FET \\
\hline$-10 q$ vs. $+20 p$ & $\mathrm{AC}$ & 0.014 & FET \\
\hline+20 p vs. $+20 q$ & $\mathrm{AC}$ & 0.003 & FET \\
\hline
\end{tabular}

Individual imbalances vs.

clinicopathological factors

$\begin{array}{llll}-1 \text { p vs. +EGFR } & \text { OD } & 0.025 & \text { WT } \\ -1 \text { p vs. }- \text { p53 } & \text { OD } & 0.049 & \text { WT } \\ +7 q \text { vs. +p53 } & \text { OD } & 0.039 & \text { WT } \\ +7 \text { p vs. +EGFR } & \text { AC } & 0.046 & \text { WT } \\ -10 \text { p vs. +EGFR } & \text { AC } & 0.024 & \text { WT } \\ +7 \text { p vs. short-term survival } & \text { AC } & 0.009 & \text { LR } \\ -10 \text { q vs. short-term survival } & \text { AC } & 0.039 & \text { LR }\end{array}$

Clinicopathological factors vs.

clinicopathological factors

\begin{tabular}{llll}
+ MIB 1 & AC & 0.045 & FET \\
+ p53 & AC & 0.031 & WT \\
+ p53 vs. - EGFR & AC & 0.046 & KW \\
\hline
\end{tabular}

${ }^{\mathrm{a} O D}$, oligodendrogliomas; AC, astrocytomas. ${ }^{\mathrm{b}} \mathrm{FET}$, Fisher exact test; WT, Wilcoxon test; LR, log-rang test; KW, Kruskal-Wallis test.

17.0 month (range, 1-72 months, \pm 23.5 ) in the astrocytic group. Seven of 11 patients in the oligodendroglial and 5 of 23 patients in the astrocytic group were alive at the end of follow-up period.

Comparative genomic hybridization. Eleven of 14 oligodendrogliomas and 20 of 23 astrocytomas revealed chromosomal aberrations (Fig. 1A and B; Table I). The average number of chromosomal aberrations was 4.8 (range, $0-13 ; \mathrm{SD} \pm 3.8$ ) in oligodendrogliomas and 5.7 (range, 0-16; $\mathrm{SD} \pm 4.4$ ) in astrocytomas. In both, oligodendrogliomas and astrocytomas, the mean number of gains (2.3 and 3.1, respectively) was similar to the mean number of losses (2.5 and 2.6). However, the pattern of genomic imbalances differed between the groups. Whereas $+7 \mathrm{q}(43 \%),-1 \mathrm{p}(36 \%)$, and $-4 \mathrm{q}(29 \%)$ were the most frequent imbalances in oligodendrogliomas, $+7 \mathrm{q}(57 \%),-10 \mathrm{q}$ $(52 \%),+7 \mathrm{p}(48 \%),-9 \mathrm{p}(43 \%),-10 \mathrm{p}(35 \%),+20 \mathrm{q}(30 \%)$, and $+20 \mathrm{p}(26 \%)$, were the most common imbalances in astrocytomas. Recurrent high-level amplifications encompassing the $7 \mathrm{p} 11$ region were observed in a tumor evolving from astrocytoma II to GBM (cases G1 and G37 R) and in 3 other GBM cases. Fig. 2 depicts a CGH karyogram and ideogram of case G22 including a proximal 7p amplification.

Immunohistochemistry. Nuclear p53 overexpression was detected in 5 (4 score 2, 1 score 3 ) oligodendrogliomas and 17 (13 score 2, 4 score 3 ) astrocytomas. Pronounced nuclear expression of PTEN was revealed in 4 (2 score 2, 2 score 3 ) oligodendrogliomas and 8 (2 score 2, 6 score 3 ) astrocytomas. Elevated membranous EGFR expression was noted for 6 (4 score 2, 2 score 3 ) oligodendrogliomas and 11 (5 score 2 , 6 score 3 ) astrocytomas and MIB-1 positivity in at least $5 \%$ of the cells was recorded for 4 oligodendrogliomas and 15 astrocytomas. Fig. 3 depicts representative microscopic images of EGFR, PTEN, and p53 overexpression in astrocytic cases.

Correlation of CGH and clinicopathological data. Some of the most common individual imbalances correlated significantly with the increasing number of overall imbalances, that were $+7 \mathrm{q}$ in the oligodendroglial $(\mathrm{p}=0.026)$ as well as in the astrocytic group $(\mathrm{p}=0.001)$ and furthermore, $-9 \mathrm{p}(\mathrm{p}=0.001)$, $-10 q(p=0.001),+20 p(p=0.007)$, and $+20 q(p<0.001)$ in the astrocytic group (Table II). Several individual imbalances in the astrocytic group showed a combined appearance, that were $+7 \mathrm{p},+7 \mathrm{q},-10 \mathrm{p}$, and $-10 \mathrm{q}$ in any dual combination $(\mathrm{p}<0.05)$ and furthermore, $+7 \mathrm{q} /+20 \mathrm{p}(\mathrm{p}=0.019),-9 \mathrm{p} /-10 \mathrm{q}(\mathrm{p}=0.036)$, $-9 \mathrm{p} /+20 \mathrm{q}(\mathrm{p}<0.001),-10 \mathrm{q} /+20 \mathrm{p}(\mathrm{p}=0.014)$, and $+20 \mathrm{p} /+20 \mathrm{q}$ $(\mathrm{p}=0.003)$. Among IHC markers, $\mathrm{p} 53$ overexpression correlated positively with $+7 \mathrm{q}(\mathrm{p}=0.039)$ and negatively with $-1 \mathrm{p}$ $(\mathrm{p}=0.049)$ in the oligodendroglial group. Moreover, increased EGFR expression correlated positively with $-1 \mathrm{p}(\mathrm{p}=0.025)$ in the oligodendroglial and with $+7 \mathrm{p}(\mathrm{p}=0.046)$ and $-10 \mathrm{p}$ $(p=0.024)$ in the astrocytic group. In general, MIB-1 $(p=0.045)$ and $\mathrm{p} 53(\mathrm{p}=0.031)$ were more highly expressed in astrocytomas than in oligodendrogliomas, and p53 and EGFR expression was inversely correlated $(\mathrm{p}=0.046)$ within the astrocytic group. The log-rank test disclosed comparably shorter overall survival rates for astrocytic cases implemented with $+7 p(p=0.009)($ Fig. 4$)$ or with $-10 q(p=0.039)$.

\section{Discussion}

Several molecular genetic studies have established a pattern of chromosomal aberrations in gliomas which is increasingly assisting a genetically based classification scheme adjunct to the established histopathological criteria. In this respect, the present study could complement some notable results to the tumor biology of diffuse gliomas.

Oligodendrogliomas are characterized by $-1 \mathrm{p}$ or combined $-1 p /-19 q$ and these findings were a hallmark in defining a genetic basis for histological subclassfication of gliomas 
(12). Further studies could support the significance of these non-random aberrations $(13,14)$ and demonstrate a close relationship of $-1 \mathrm{p} /-19 \mathrm{q}$ with chemotherapy sensitivity (4). These correlations led to a genetic routine diagnosis for $-1 \mathrm{p} /$ $19 q$ allelic losses that predict a favourable clinical course for patients. In accordance with these findings we found $-1 \mathrm{p}$ as one of the most significant findings in oligodendrogliomas. Furthermore, we found a relationship between -1p and low expression of p53 which resembles findings of inverse correlation of $\mathrm{p} 53$ mutations and loss of heterozygosity of $1 \mathrm{p}$ $(15,16)$. It has been assumed that classic oligodendrogliomas are correlated with $-1 \mathrm{p} /-19 \mathrm{q}$ whereas oligodendrogliomas with astrocytic features are more related with p53 mutations (17). In contrast to other studies, $-19 q$ was only detected in two of our oligodendroglial cases which might be attributed to sample bias or limitation of the CGH method which often is hampered to establish a suitable profile for the critical chromosome 19 (18). FISH or LOH studies would be the methods of choice to unravel minute deletions on $19 q$ (19). Tumor heterogeneity might also account for the failure to detect more frequently $-19 q$ as for instance, only the recurrent case of G10 (not presented here) displayed $-19 q$. It should be noted that 6 of our oligodendroglial and astrocytic cases revealed no imbalances despite repeated $\mathrm{CGH}$ analysis. This holds with other $\mathrm{CGH}$ studies on gliomas which likewise reported a respective number of non-informative cases even with microdissected tumor samples (20).

We encountered most frequently $+7 \mathrm{q}$ in oligodendrogliomas; however, this imbalance correlated neither with -10 nor with $+7 \mathrm{p} 11$, that specifically targets the EGFR gene locus. Gains of 7 or $7 q$ were recurrently observed in $\mathrm{CGH}$ studies of oligodendrogliomas $(14,21,22)$ and seemingly represent an alternative pathway in oligodendroglial pathogenesis. All 11 informative oligodendrogliomas in our study were either affected by $-1 \mathrm{p}$ or $+7 \mathrm{q}$, or $-1 \mathrm{p} /+7 \mathrm{q}$. A promising target gene on $7 \mathrm{q}$ might be the CDK6, which seems to be frequently overexpressed in medulloblastomas (23) whereas for $-1 \mathrm{p} /-19 \mathrm{q}$ no classical tumor suppressor or a chemotherapysensitive target gene has been identified $(17,24)$.

The most characteristic imbalances in our astrocytic group were +7 and -10 contributing to the established pattern of genomic aberration in the astrocytic lineage (25). Gain of $7 p$ are commonly associated with amplification of the EGFR gene locus and related with EGFR immunopositivity. The significance of this relationship was also demonstrated in our astrocytic series and furthermore, $+7 \mathrm{p}$ revealed to be the most negative prognostic marker in this group, which might be of clinical relevance in view of the emerging therapeutic options targeting EGFR and EGFR signalling pathways (3). EGFR and p53 overexpression disclosed a negative association in the astrocytic group which is consistent with some studies also noting an inverse correlation of these markers, yet their assumed mutual exclusivity remains to be further clarified (3).

Amplification of 7p11 was detected only in one oligodendroglioma (case G1) which disclosed no positive EGFR immunostaining. However, the tumor recurred 20 months later as a secondary GBM (case G37 rec) which although showing essentially the same imbalances revealed increased immunopositivity for EGFR, PTEN, MIB-1, and p53. Low-grade oligodendrogliomas developing rapidly to GBM are rare (26). $\mathrm{LOH}$ of chromosome 10 is the most common aberration in high-grade gliomas and identified as a negative prognostic factor (27). Besides PTEN, other candidate genes affected by - 10 aberrations include LGII (leucine-rich gene-glioma inactivated, 1) of which the expression in malignant gliomas compared to normal brain tissue is severely reduced (28), and DMBT1 (deleted in malignant brain tumour-1), which seems to be affected in a minor set of gliomas (29). Gains of 20p and/or 20q were only mentioned marginally for their possible involvement in glial pathogenesis (30), though the putative serine/threonine kinase gene STK15 located at 20q13 has been recognized as a possible candidate gene involved in glial tumorigenesis $(31,32)$. Other common regions of deletions in astrocytomas include $-9 \mathrm{p}$. The CDKN2 locus located at 9p21 encodes for the cell cycle control genes $C D K N 2 A$ and $C D K N 2 B$ which are frequently targeted in various tumors. Recently, it has been reported that patients with high-grade gliomas characterized by $-9 p$ and $-10 q$ benefit from Temozolomide, an alkylating chemotherapeutic agent (33). Considering this, it would be a diagnostic challenge to identify those gliomas with therapeutical susceptibility depending on the otherwise negative prognostic markers $-9 \mathrm{p} /-10 \mathrm{q}$.

In summary, the present study identified significant interrelationships of $\mathrm{CGH}$ results and clinicopathological features in diffuse gliomas which in light of the emerging molecular biological influence on the clinical management of gliomas anticipate further recognition.

\section{Acknowledgements}

We thank Judit Wolf-Salgó and Christina Enders for excellent technical assistance.

\section{References}

1. Kleihues P, Louis DN, Scheithauer BW, Rorke LB, Reifenberger G, Burger PC and Cavenee WK: The WHO classification of tumors of the nervous system. J Neuropathol Exp Neurol 61: 215-225, 2002.

2. Gupta M, Djalilvand A and Brat DJ: Clarifying the diffuse gliomas: an update on the morphologic features and markers that discriminate oligodendroglioma from astrocytoma. Am J Clin Pathol 124: 755-768, 2005.

3. Kelley TW, Tubbs RR and Prayson RA: Molecular diagnostic techniques for the clinical evaluation of gliomas. Diagn Mol Pathol 14: 1-8, 2005.

4. Cairncross JG, Ueki K, Zlatescu MC, Lisle DK, Finkelstein DM, Hammond RR, Silver JS, Stark PC, Macdonald DR, Ino Y, Ramsay DA and Louis DN: Specific genetic predictors of chemotherapeutic response and survival in patients with anaplastic oligodendrogliomas. J Natl Cancer Inst 90: 1473-1479, 1998.

5. Koschny R, Koschny T, Froster UG, Krupp W and Zuber MA: Comparative genomic hybridization in glioma: a meta-analysis of 509 cases. Cancer Genet Cytogenet 135: 147-159, 2002.

6. Besson A and Yong VW: Mitogenic signaling and the relationship to cell cycle regulation in astrocytomas. J Neurooncol 51: 245-264, 2001.

7. Romeike BF, Jung V, Feiden W, Moringlane JR, Zang KD and Urbschat SM: Distribution of epidermal growth factor receptor protein correlates with gain in chromosome 7 revealed by comparative genomic hybridization after microdissection in glioblastoma multiforme. Pathol Res Pract 197: 427-431, 2001.

8. Baeza N, Weller M, Yonekawa Y, Kleihues P and Ohgaki H: PTEN methylation and expression in glioblastomas. Acta Neuropathol (Berl) 106: 479-485, 2003. 
9. Sano T, Lin H, Chen X, Langford LA, Koul D, Bondy ML, Hess KR, Myers JN, Hong YK, Yung WK and Steck PA: Differential expression of MMAC/PTEN in glioblastoma multiforme: relationship to localization and prognosis. Cancer Res 59: 1820-1824, 1999.

10. Watanabe K, Tachibana O, Sata K, Yonekawa Y, Kleihues P and Ohgaki H: Overexpression of the EGF receptor and p53 mutations are mutually exclusive in the evolution of primary and secondary glioblastomas. Brain Pathol 6: 217-223, 1996.

11. Gunawan B, Schulten HJ, von Heydebreck A, Schmidt B, Enders C, Hoer J, Langer C, Schuler P, Schindler CG, Kuhlgatz J and Fuzesi L: Site-independent prognostic value of chromosome $9 \mathrm{q}$ loss in primary gastrointestinal stromal tumours. J Pathol 202: 421-429, 2004.

12. Reifenberger J, Reifenberger G, Liu L, James CD, Wechsler W and Collins VP: Molecular genetic analysis of oligodendroglial tumors shows preferential allelic deletions on $19 q$ and $1 \mathrm{p}$. Am J Pathol 145: 1175-1190, 1994.

13. Burger PC, Minn AY, Smith JS, Borell TJ, Jedlicka AE, Huntley BK, Goldthwaite PT, Jenkins RB and Feuerstein BG: Losses of chromosomal arms $1 \mathrm{p}$ and $19 \mathrm{q}$ in the diagnosis of oligodendroglioma. A study of paraffin-embedded sections. Mod Pathol 14: 842-853, 2001.

14. Kitange G, Misra A, Law M, Passe S, Kollmeyer TM, Maurer M, Ballman K, Feuerstein BG and Jenkins RB: Chromosomal imbalances detected by array comparative genomic hybridization in human oligodendrogliomas and mixed oligoastrocytomas. Genes Chromosomes Cancer 42: 68-77, 2005.

15. Ino Y, Betensky RA, Zlatescu MC, Sasaki H, Macdonald DR, Stemmer-Rachamimov AO, Ramsay DA, Cairncross JG and Louis DN: Molecular subtypes of anaplastic oligodendroglioma: implications for patient management at diagnosis. Clin Cancer Res 7: 839-845, 2001.

16. Ueki K, Nishikawa R, Nakazato Y, Hirose T, Hirato J, Funada N, Fujimaki T, Hojo S, Kubo O, Ide T, Usui M, Ochiai C, Ito S, Takahashi H, Mukasa A, Asai A and Kirino T: Correlation of histology and molecular genetic analysis of 1p, 19q, 10q, TP53, EGFR, CDK4, and CDKN2A in 91 astrocytic and oligodendroglial tumors. Clin Cancer Res 8: 196-201, 2002.

17. Ueki K: Oligodendroglioma: impact of molecular biology on its definition, diagnosis and management. Neuropathology 25: 247-253, 2005

18. Solinas-Toldo S, Wallrapp C, Muller-Pillasch F, Bentz M, Gress T and Lichter P: Mapping of chromosomal imbalances in pancreatic carcinoma by comparative genomic hybridization. Cancer Res 56: 3803-3807, 1996.

19. Smith JS, Alderete B, Minn Y, Borell TJ, Perry A, Mohapatra G, Hosek SM, Kimmel D, O'Fallon J, Yates A, Feuerstein BG, Burger PC, Scheithauer BW and Jenkins RB: Localization of common deletion regions on $1 \mathrm{p}$ and $19 \mathrm{q}$ in human gliomas and their association with histological subtype. Oncogene 18: 4144-4152, 1999

20. Hirose Y, Aldape KD, Chang S, Lamborn K, Berger MS and Feuerstein BG: Grade II astrocytomas are subgrouped by chromosome aberrations. Cancer Genet Cytogenet 142: 1-7, 2003.

21. Bigner SH, Matthews MR, Rasheed BK, Wiltshire RN, Friedman HS, Friedman AH, Stenzel TT, Dawes DM, McLendon RE and Bigner DD: Molecular genetic aspects of oligodendrogliomas including analysis by comparative genomic hybridization. Am J Pathol 155: 375-386, 1999.
22. Kros JM, van Run PR, Alers JC, Beverloo HB, van den Bent MJ, Avezaat CJ and van Dekken H: Genetic aberrations in oligodendroglial tumours: an analysis using comparative genomic hybridization (CGH). J Pathol 188: 282-288, 1999.

23. Mendrzyk F, Radlwimmer B, Joos S, Kokocinski F, Benner A, Stange DE, Neben K, Fiegler H, Carter NP, Reifenberger G, Korshunov A and Lichter P: Genomic and protein expression profiling identifies CDK6 as novel independent prognostic marker in medulloblastoma. J Clin Oncol 23: 8853-8862, 2005.

24. Jeuken JW, von Deimling A and Wesseling P: Molecular pathogenesis of oligodendroglial tumors. J Neurooncol 70: 161-181, 2004.

25. Wiltshire RN, Herndon JE II, Lloyd A, Friedman HS, Bigner DD, Bigner SH and McLendon RE: Comparative genomic hybridization analysis of astrocytomas: prognostic and diagnostic implications. J Mol Diagn 6: 166-179, 2004.

26. Rosso SM, van Dekken H, Krishnadath KK, Alers JC and Kros JM: Detection of chromosomal changes by interphase cytogenetics in biopsies of recurrent astrocytomas and oligodendrogliomas. J Neuropathol Exp Neurol 56: 1125-1131, 1997.

27. Balesaria S, Brock C, Bower M, Clark J, Nicholson SK, Lewis P, de Sanctis S, Evans H, Peterson D, Mendoza N, Glaser MG, Newlands ES and Fisher RA: Loss of chromosome 10 is an independent prognostic factor in high-grade gliomas. Br J Cancer 81: 1371-1377, 1999

28. Chernova OB, Somerville RP and Cowell JK: A novel gene, LGI1, from 10q24 is rearranged and downregulated in malignant brain tumors. Oncogene 17: 2873-2881, 1998.

29. Somerville RP, Shoshan Y, Eng C, Barnett G, Miller D and Cowell JK: Molecular analysis of two putative tumour suppressor genes, PTEN and DMBT, which have been implicated in glioblastoma multiforme disease progression. Oncogene 17: $1755-1757,1998$.

30. Brunner C, Jung V, Henn W, Zang KD and Urbschat S: Comparative genomic hybridization reveals recurrent enhancements on chromosome 20 and in one case combined amplification sites on $15 \mathrm{q} 24 \mathrm{q} 26$ and $20 \mathrm{p} 11 \mathrm{p} 12$ in glioblastomas. Cancer Genet Cytogenet 121: 124-127, 2000.

31. Reichardt W, Jung V, Brunner C, Klein A, Wemmert S, Romeike BF, Zang KD and Urbschat S: The putative serine/ threonine kinase gene STK15 on chromosome 20q13.2 is amplified in human gliomas. Oncol Rep 10: 1275-1279, 2003.

32. Klein A, Reichardt W, Jung V, Zang KD, Meese E and Urbschat S: Overexpression and amplification of STK15 in human gliomas. Int J Oncol 25: 1789-1794, 2004.

33. Wemmert S, Ketter R, Rahnenfuhrer J, Beerenwinkel N, Strowitzki M, Feiden W, Hartmann C, Lengauer T, Stockhammer F, Zang KD, Meese E, Steudel WI, von Deimling A and Urbschat S: Patients with high-grade gliomas harboring deletions of chromosomes $9 p$ and $10 q$ benefit from temozolomide treatment. Neoplasia 7: 883-893, 2005. 